\title{
Activation of Human Neutrophil Nicotinamide Adenine Dinucleotide Phosphate, Reduced (Triphosphopyridine Nucleotide, Reduced) Oxidase by Arachidonic Acid in a Cell-free System
}

John T. Curnutte

Department of Pediatrics and Communicable Diseases, Section of Pediatric Hematology/Oncology, The University of Michigan Medical School, Ann Arbor, Michigan 48109

\begin{abstract}
Sonicates from unstimulated human neutrophils produce no measurable superoxide since the superoxide-generating enzyme, NADPH oxidase, is inactive in these preparations. Previous attempts to activate the oxidase in disrupted cells with conventional neutrophil stimuli have been unsuccessful. This report describes a cell-free system in which arachidonic acid $(82 \mu \mathrm{M})$ was able to activate superoxide generation that was dependent upon the presence of NADPH and the sonicate. For activation to occur, both the particulate and supernatant fractions of the sonicate must be present. Calcium ions, which are required for activation of intact neutrophils by arachidonate, were not necessary in the cell-free system. In quantitative terms, the superoxide-generating activity in the cell-free system could account for at least $20-50 \%$ of the superoxide rate observed in intact neutrophils stimulated with arachidonate.

Sonicates from patients with chronic granulomatous disease (CGD) could not be activated by arachidonic acid in the cellfree system. In three patients representing both genetic forms of CGD, the defect appeared to reside in the particulate fraction. The soluble cofactor was normal in all three patients and could be used to activate normal neutrophil pellets in the presence of arachidonic acid. Thus, at least a portion of the activation mechanism in the neutrophil, that residing in the soluble phase, appeared to be normal in patients with CGD.
\end{abstract}

\section{Introduction}

When neutrophils phagocytize opsonized microorganisms, they exhibit a several 100 -fold increase in oxygen consumption with concomitant superoxide generation $(1,2)$. The central event in the activation of this respiratory burst is the conversion of a membrane-bound NADPH oxidase from an inactive to an active form (1). Once activated, the oxidase can catalyze the one-electron reduction of oxygen to superoxide.

The molecular details of how NADPH oxidase is activated have not been established. Attempts to study this problem at

This work was presented at the National Meeting of the American Society of Hematology, Miami Beach, Florida, 1-4 December 1984, and was published in abstract form in 1984. Blood. 64:66.

Address correspondence to Dr. Curnutte.

Received for publication 21 December 1984 and in revised form 7 February 1985.

J. Clin. Invest.

(c) The American Society for Clinical Investigation, Inc.

0021-9738/85/05/1740/04\$1.00

Volume 75, May 1985, 1740-1743 the intact cell level have been frustrated by the complexity of the biochemical and cellular changes that occur during phagocytosis. Ideally, NADPH oxidase activation would best be approached using a cell-free system in which the constituents of the activation reaction could be carefully controlled. For reasons that are not clear, however, it has not been possible to activate the oxidase once unstimulated neutrophils have been disrupted. Recently, arachidonic acid has been found to be a potent stimulus of the respiratory burst (3-5) in intact cells. This agent has the unique property of causing activation of the respiratory burst without the lag time characteristic of other neutrophil stimuli, suggesting that arachidonic acid might enter the activation pathway at some distal point and might, therefore, activate the oxidase in disrupted neutrophils.

This report describes a cell-free system in which NADPH oxidase from unstimulated human neutrophils was activated by arachidonic acid. In addition, the effect of arachidonic acid on disrupted neutrophils from patients with chronic granulomatous disease (CGD) ${ }^{1}$ is reported. Concurrent with the development of the cell-free system in this laboratory, both McPhail et al. (6) and Bromberg and Pick (7) have likewise been able to activate NADPH oxidase with arachidonate using human neutrophils and guinea pig peritoneal macrophages, respectively.

\section{Methods}

Chemicals. Distilled deionized water was used throughout all experiments and was obtained using an ultra-pure water filter (Barnstead Co., Sybron Corp., Boston, MA). Arachidonic acid was obtained in the free acid form from Nu-Chek Prep., Inc., Elysian, MN. Ferricytochrome c (type VI), superoxide dismutase (SOD) from bovine blood, and NADPH (type X) were obtained from Sigma Chemical Co., St. Louis, MO. Macrodex (dextran 70) and Ficoll-Paque were products of Pharmacia Fine Chemicals, Div. of Pharmacia Inc., Piscataway, NJ.

Preparation of neutrophils. Human neutrophils were purified from whole blood by combining the dextran sedimentation method of Skoog and Beck (8) with a Ficoll-Paque gradient procedure that is outlined in detail elsewhere (9). This procedure resulted in cell preparations that contained $\geq 98 \%$ neutrophils that had a viability $\geq 98 \%$.

Disruption of neutrophils. Neutrophils were suspended in phosphatebuffered saline (PBS) (138 mM NaCl, $2.7 \mathrm{mM} \mathrm{KCl}, 16.2 \mathrm{mM}$ $\mathrm{Na}_{2} \mathrm{HPO}_{4}$, and $1.47 \mathrm{mM} \mathrm{KH}_{2} \mathrm{PO}_{4}$ ) at a concentration of $10^{8}$ cells $/ \mathrm{ml}$. The cells were disrupted by sonication for $40 \mathrm{~s}$ while immersed in an ice-water bath using the microtip probe of a sonicator (Artek Systems Corp., Dynatech Corp., Farmingdale, NY) set at 35\% maximal energy. Greater than $98 \%$ of the cells were disrupted by this technique. The

1. Abbreviations used in this paper: CGD, chronic granulomatous disease; SOD, superoxide dismutase. 
few remaining intact cells and undisrupted nuclei were removed by centrifugation at $250 \mathrm{~g}$ at $4^{\circ} \mathrm{C}$ for $4 \mathrm{~min}$. The supernate from this centrifugation was termed "whole sonicate" and was used within $3 \mathrm{~h}$.

Preparation of sonicate fractions. For certain experiments, the whole sonicate was separated into pellet and supernate fractions by centrifugation at $50,000 \mathrm{~g}$ for $90 \mathrm{~min}$ at $4^{\circ} \mathrm{C}$. The clear supernate was immediately removed and stored on ice. The pellet was resuspended in ice-cold PBS (without calcium and magnesium) by vigorous vortexing and sonication as described above for intact neutrophils. The supernate fraction contained the soluble components from neutrophils at a concentration of $10^{8}$ cell equivalents $/ \mathrm{ml}$. Similarly, the pellet fraction contained $10^{8}$ cell equivalents $/ \mathrm{ml}$.

Preparation of arachidonic acid. Stock solutions of arachidonic acid $(2.5 \mathrm{mg} / \mathrm{ml}$ in a $25 \% \mathrm{vol} / \mathrm{vol}$ aqueous solution of ethanol) were prepared immediately before use as described elsewhere (3).

Superoxide release. Superoxide release from neutrophils was measured continuously at $25^{\circ} \mathrm{C}$ by following the SOD-inhibitable reduction of ferricytochrome $c$ at $550 \mathrm{~nm}$. The standard assay mixture $(1.0 \mathrm{ml})$ consisted of $65 \mathrm{mM}$ potassium phosphate buffer (pH 7.0) containing $0.075 \mathrm{mM}$ ferricytochrome $\mathrm{c}$ and $0.16 \mathrm{mM}$ NADPH. The reference cuvette contained $60 \mu \mathrm{g} / \mathrm{ml}$ of SOD. Neutrophil sonicates (or the corresponding supernates and pellets) were present as indicated in the legends to the tables. Reaction rates were measured for $3 \mathrm{~min}$ before the addition of arachidonic acid (final concentration, $82 \mu \mathrm{M}$ ), which was used to initiate superoxide production. Initial maximal rates were calculated for each reaction from the continuous tracings obtained on a dual beam spectrophotometer (Uvikon 810; Kontron Analytical, Zurich, Switzerland).

\section{Results}

Activation of unstimulated neutrophil sonicates with arachidonic acid. Table I shows the effect of arachidonic acid on superoxide generation by neutrophil sonicates. Before the addition of arachidonate, no superoxide generation was measured. However, within several seconds after the addition of arachidonic acid, a substantial rate of superoxide production was noted that continued at a linear rate until all of the NADPH was consumed (data not shown). This result indicated that arachi-

Table I. Activation of Neutrophil Sonicates by Arachidonic Acid

\begin{tabular}{lll}
\hline & \multicolumn{2}{l}{$\mathrm{O}_{2}^{-}$generation } \\
\cline { 2 - 3 } Reaction mixture & $\begin{array}{l}\text { Without } \\
\text { arachidonate }\end{array}$ & $\begin{array}{l}\text { With } \\
\text { arachidonate }\end{array}$ \\
\hline & $n m o l / m i n$ & $n m o l / m i n$ \\
Complete reaction mixture & 0 & $1.45 \pm 0.35(\mathrm{SD})$ \\
Substitute pellet for sonicate & 0 & $0.01 \pm 0.01$ \\
Substitute supernate for sonicate & 0 & $0.02 \pm 0.05$ \\
Sonicate pellet plus supernate & 0 & $1.39 \pm 0.26$ \\
Sonicate pellet plus supernate $(\times 2.5)$ & 0 & $2.45 \pm 0.41$ \\
\hline
\end{tabular}

Superoxide production was measured as described in Methods both before and after the addition of $82 \mu \mathrm{M}$ arachidonic acid. Initial maximal velocities are reported. Reaction mixtures contained $100 \mu \mathrm{l}$ of whole sonicate (after removal of intact cells and nuclei) derived from $10^{7}$ neutrophils and prepared as described in Methods. Where indicated, $100 \mu \mathrm{l}$ of sonicate pellet or supernate (each derived from $10^{7}$ cells and prepared at 50,000 $\times 90 \mathrm{~min}$ ) was substituted for the whole sonicate. In the experiments in which pellet and supernate were mixed to reconstitute the sonicate, $100 \mu \mathrm{l}$ of pellet was mixed with either $100 \mu$ l supernate or $250 \mu$ l supernate (indicated by $\times 2.5$ ). Each rate is reported as the mean $\pm \mathrm{SD}$ of four experiments. donic acid could activate superoxide production in the neutrophil sonicate in a manner similar to that observed with intact resting neutrophils.

Since activated NADPH oxidase has been shown to reside in the plasma membrane of neutrophils $(10,11)$, an experiment was performed to see whether the superoxide-generating activity observed in the cell-free system was also present in the pellet fraction. Sonicates were spun at $50,000 \mathrm{~g}$, and the resulting supernates and pellets were assayed separately. As the data in Table I show, the pellet by itself was not activated with arachidonic acid. Similarly, the supernate fraction alone was inactive. Activity could be reconstituted, however, when the sonicate pellet and supernate were mixed together, indicating that the oxidase components had not been denatured by the centrifugation procedure. Interestingly, when $2^{1 / 2}$ times as much supernate was added to a fixed amount of sonicate pellet, there was an enhancement in the rate of superoxide production, indicating a dose dependency on some constituent of the supernate fraction.

Using the reconstituted sonicate preparation in which the supernate fraction was $2 \frac{1}{2}$-fold enriched, a series of control experiments were performed. The results of these are shown in Table II. Omission of either arachidonate or NADPH from the reaction mixture resulted in a failure to detect superoxide. When both the supernate and pellet fractions were omitted, there was no superoxide production, thus establishing that the generation of superoxide was not due to some side reaction involving arachidonic acid and NADPH. When either the supernate fraction or the pellet fraction was boiled for 10 min., there was a $>95 \%$ loss of activity, showing that the activation components of both fractions were heat labile.

Changes in intracellular concentrations of ionized calcium have been proposed to play a role in the regulation of oxidase activity $(12-14)$. Since the experiments described above were all performed with buffers and reagents prepared without calcium, the results suggest that activation of the oxidase does not require calcium when arachidonic acid is the stimulus. To exclude more rigorously the involvement of calcium in the activation process, $10 \mathrm{mM}$ EGTA was incorporated into both the PBS used for cell disruption and the buffers used in the superoxide assay. As shown in Table II, the presence of 10 mM EGTA had no inhibitory effect on oxidase activation.

Table II. Activation of Reconstituted Sonicates by Arachidonic Acid

\begin{tabular}{ll}
\hline Reaction mixture & $\mathrm{O}_{2}^{-}$generation \\
\hline & $n$ mol/min \\
Complete mixture & $2.81 \pm 0.46(\mathrm{SD})$ \\
Omit arachidonate & $0.01 \pm 0.02$ \\
Omit NADPH & $0.01 \pm 0.02$ \\
Omit both supernate and pellet fractions & 0 \\
Boiled supernate fraction with untreated pellet & 0 \\
Boiled pellet fraction with untreated supernate & $0.12 \pm 0.23$ \\
Complete mixture plus EGTA $(10 \mathrm{mM})$ & $2.41 \pm 0.32$
\end{tabular}

Superoxide generation was measured as described in Methods with each reaction mixture containing $50 \mu$ l sonicate pellet (from $5 \times 10^{6}$ cells) and $250 \mu \mathrm{l}$ sonicate supernate (from $2.5 \times 10^{7}$ cells). Where indicated, sonicate fractions were heated in boiling water for $10 \mathrm{~min}$. Each result is expressed as the mean $\pm \mathrm{SD}$ of four experiments. 
To exclude the possibility that small amounts of superoxide were initiating an arachidonate-catalyzed free radical chain reaction resulting in greatly amplified superoxide rates, the effect of SOD on NADPH oxidation by the sonicate was tested. In the absence of SOD, reaction mixtures containing both supernate and pellet fractions exhibited a marked increase in the rate of NADPH oxidation, from $0.25 \pm 0.08$ to $3.78 \pm 0.63$ $\mathrm{nmol} / \mathrm{min}$ per $10^{7}$ cell equivalents $(n=3)$ following the addition of $82 \mu \mathrm{M}$ arachidonate. ${ }^{2}$ The addition of SOD $(60 \mu \mathrm{g} / \mathrm{ml})$ caused only a small decrease $(<15 \%)$ in rates of NADPH oxidation in both cases, indicating that superoxide anion $\left(\mathrm{O}_{2}^{-}\right)$was simply a product of the reaction and not a catalyst for NADPH oxidation.

Recovery of NADPH oxidase activity in neutrophil sonicates. Intact human neutrophils stimulated with arachidonate generate superoxide at a rate of $105 \pm 24 \mathrm{SD} \mathrm{nM} \mathrm{O}-1 / \mathrm{min}$ per $10^{7}$ cells (4). Ideally, the superoxide-generating activity recovered in the cell-free system should account for a large percentage of this intact cell rate. Using the data in Table II, one can calculate that the complete reaction mixture generates superoxide at a rate of $5.62 \mathrm{nmol} \mathrm{O}_{2}^{-} / \mathrm{min}$ per $10^{7}$ cell equivalents, a rate which is $\sim 5 \%$ that observed in the intact cell. However, when the effect of pellet concentration on superoxide rate was examined, it was found that the amount of pellet protein used in the cell-free assays was saturating. When the amount of pellet protein was reduced to $1-5 \mu \mathrm{l} / \mathrm{ml}$ of reaction mixture (equivalent to $1-5 \times 10^{5}$ cells), a linear relationship between pellet protein and superoxide rates was obtained (data not shown). When superoxide rates were then calculated under these conditions, rates in the cell-free system ranged between 21 and $46 \mathrm{nmol} \mathrm{O}_{2}^{-} / \mathrm{min}$ per $10^{7}$ cell equivalents, thus accounting for $\sim 20-50 \%$ of the intact cell superoxide rate.

Superoxide generation by sonicate fractions from normal and CGD neutrophils. Neutrophils from CGD patients fail to undergo a respiratory burst in response to all known neutrophil stimuli. The failure to activate the respiratory burst in CGD is believed to be due either to a defective NADPH oxidase or to a defective activation pathway. It was of interest to examine the sonicates from CGD neutrophils for two reasons. First, failure to detect superoxide production in the cell-free system would provide confirmatory evidence that arachidonate does, in fact, activate NADPH oxidase to make superoxide. Second, it would be possible to determine whether the soluble component in the supernate was defective in CGD or whether the pellet material was abnormal (or both). The results of these experiments are shown in Table III. In these studies, supernate from either normal or CGD neutrophils was mixed in different combinations with pellet material from normal and CGD cells. Three different CGD patients were examined in these experiments, two of whom had the X-linked form of CGD, while the third patient had the autosomal recessive form. The control mixture, in which normal supernate and pellet were mixed together, showed the expected rate of superoxide generation. When both the supernate and pellet fractions were derived from CGD neutrophils, no superoxide was generated.

2. Reaction mixtures for these experiments were similar to those described in Table II except that cytochrome $c$ was omitted, NADPH was present only in the sample cuvette, and SOD (when tested) was present in both cuvettes. Reaction rates were measured at $340 \mathrm{~nm}$. Neither the pellet nor supernate fraction alone oxidized NADPH in the presence of arachidonate.
Table III. Superoxide Generation by Sonicate Fractions from Normal and CGD Neutrophils

\begin{tabular}{lll}
\hline \multicolumn{2}{l}{ Source of sonicate fraction } & \\
\cline { 2 - 2 } Supernate & Pellet & $\mathrm{O}_{2}^{-}$generation \\
\hline & & $n$ mol/min \\
Normal & Normal & $2.70 \pm 0.11(\mathrm{SD})$ \\
CGD & CGD & $0 \pm 0$ \\
Normal & CGD & $0 \pm 0$ \\
CGD & Normal & $2.11 \pm 0.65$ \\
\hline
\end{tabular}

Superoxide generation was measured as described in Methods. Each reaction mixture contained $50 \mu$ of pellet fraction derived from 5 $\times 10^{6}$ neutrophils and $250 \mu$ of supernate fraction derived from 2.5 $\times 10^{7}$ cells in the combinations indicated in the table. The data are expressed as the mean \pm SD from three separate experiments, each using a different CGD patient.

Thus the defect at the intact cell level was mimicked. When normal supernate was mixed with CGD pellet material, no superoxide was again detected. In contrast, supernate fraction from CGD cells was able to supply the necessary component to a normal pellet, so that normal rates of superoxide were obtained. These cross-mixing experiments indicate that the defective portion of the CGD sonicate resides in the pellet and that the soluble component required for activation is normal in CGD.

\section{Discussion}

The superoxide-generating system activated by arachidonic acid in these experiments is likely to be NADPH oxidase. Superoxide generation by residual intact cells was excluded by using vigorous sonication and low speed centrifugation spin to remove all intact cells. Similarly, noncellular side reactions involving NADPH and arachidonic acid were excluded by the control experiments. Furthermore, neither the supernate nor the particulate fraction alone could catalyze superoxide generation in the presence of both arachidonic acid and NADPH. The possibility that the large rates of superoxide production represent an amplification of trivial rates of superoxide production by means of an arachidonate-catalyzed free radical chain reaction has likewise been ruled out. Finally, the failure of pellet material from three patients with CGD to generate superoxide further establishes the superoxide generating system in the cell-free preparation as the same entity missing in CGD, namely NADPH oxidase. The data reported by McPhail et al. (6) and by Bromberg and Pick (7) further support the belief that arachidonic acid activates NADPH oxidase. These investigators have concurrently found that arachidonate can activate superoxide generation in cell-free systems from human neutrophils and guinea pig peritoneal macrophages. These reports show that the Michaelis constants for NADPH and NADH in the cell-free system are strikingly similar to those reported for NADPH oxidase, and that other fatty acids known to activate superoxide production in intact neutrophils can cause similar activation in the cell-free system.

The rate of superoxide generation in the cell-free system reported here can account for $20-50 \%$ of the superoxide rate seen in intact cells stimulated with arachidonic acid. It is 
important to point out that these figures represent minimal estimates, since the cell-free activation system has not yet been optimized. In particular, the concentration of the soluble cofactor in this system is still limiting. This is not surprising since the final dilution of all cytoplasmic components in each reaction mixture was $\sim 100$-fold compared with their concentrations in intact cells. The increase in superoxide generation observed when the amount of supernate was increased by $2 \frac{1}{2}-$ fold undoubtedly reflects a decrease in this dilution factor to 1:40. Further increases in the concentration of the soluble component may result in even greater rates of superoxide production in the cell-free system.

The experiments with the CGD neutrophils failed to disclose any heterogeneity in this disorder, despite the fact that two different genetic forms of the disease were analyzed. In all cases the soluble component was normal and the particulate material was abnormal. This result suggests that the mutations in both forms of the disease affect some aspect of the membrane. The possibility still remains, however, that these mutations could affect either the oxidase molecule itself or components of the activation pathway that reside in the membrane.

\section{Acknowledgments}

This work was supported in part by a grant from the U. S. Public Health Service, National Institutes of Health (grant 1 ROI AI2132001). Additional funding was provided by a faculty grant from the Horace H. Rackham School of Graduate Studies at the University of Michigan and from the Michigan Memorial-Phoenix Project. This work was also supported in part by institutional research grant IN40X, awarded to the University of Michigan by the American Cancer Society, and by a Starter Research Grant provided by the Society for Pediatric Research.

\section{References}

1. Babior, B. M. 1984. The respiratory burst of phagocytes. J. Clin. Invest. 73:599-601.

2. Badwey, J. A., J. T. Curnutte, J. M. Robinson, J. K. Lazdins, R. T. Briggs, M. J. Karnovsky, and M. L. Karnovsky. 1980. Comparative aspects of oxidative metabolism of neutrophils from human blood and guinea pig peritonea: magnitude of the respiratory burst, dependence upon stimulating agents, and localization of the oxidases. J. Cell. Physiol. 105:541-551.

3. Badwey, J. A., J. T. Curnutte, and M. L. Karnovsky. 1981. Cispolyunsaturated fatty acids induce high levels of superoxide production by human neutrophils. J. Biol. Chem. 256:12640-12643.

4. Curnutte, J. T., J. A. Badwey, J. M. Robinson, M. J. Karnovsky, and M. L. Karnovsky. 1984. Studies on the mechanism of superoxide release from human neutrophils stimulated with arachidonate. J. Biol. Chem. 259:11851-11857.

5. Badwey, J. A., J. T. Curnutte, J. M. Robinson, C. B. Berde, M. J. Karnovsky, and M. L. Karnovsky. 1984. Effects of free fatty acids on release of superoxide and on change of shape by human neutrophils. Reversibility by albumin. J. Biol. Chem. 259:7870-7877.

6. McPhail, L. C., C. C. Clayton, and R. Snyderman. 1984. Evidence that activation of human neutrophil NADPH oxidase involves association of a cytosolic factor with membrane components. Clin. Res. 32:315a.

7. Bromberg, Y., and E. Pick. 1984. Unsaturated fatty acids stimulate NADPH-dependent superoxide production by cell-free system derived from macrophages. Cell. Immunol. 88:213-221.

8. Skoog, W. A., and W. S. Beck. 1956. Studies on the fibrinogen, dextran, and phytohemagglutinin methods of isolating leukocytes. Blood. 11:436-454.

9. Badwey, J. A., J. T. Curnutte, C. B. Berde, and M. L. Karnovsky. 1982. Cytochalasin $\mathrm{E}$ diminishes the lag phase in the release of superoxide by human neutrophils. Biochem. Biophys. Res. Commun. 106:170-174.

10. Briggs, R. T., D. B. Drath, M. L. Karnovsky, and M. J. Karnovsky. 1975. Localization of NADH oxidase on the surface of human polymorphonuclear leukocytes by a new cytochemical method. J. Cell. Biol. 67:566-586.

11. Dewald, B., M. Baggiolini, J. T. Curnutte, and B. M. Babior. 1979. Subcellular localization of the superoxide-forming enzyme in human neutrophils. J. Clin. Invest. 63:21-29.

12. Romeo, D., G. Zabucchi, N. Miani, and F. Rossi. 1975. Ion movement across leukocyte plasma membranes and excitation of their metabolism. Nature (Lond.). 253:542-544.

13. Smolen, J. E., H. M. Korchak, and G. Weissmann. 1981. The roles of extracellular and intracellular calcium in lysosomal enzyme release and superoxide anion generation by human neutrophils. Biochim. Biophys. Acta. 677:512-520.

14. Pozzan, T., D. P. Lew, C. B. Wollheim, and R. Y. Tsien. 1983. Is cytosolic ionized calcium regulating neutrophil activation? Science (Wash. DC). 221:1413-1415. 